\title{
SIMPLIFIED MODEL OF THERMAL INTERACTIONS BETWEEN ENVIRONMENT, PROTECTIVE CLOTHING AND SKIN TISSUE
}

\author{
Bohdan Mochnacki ${ }^{1}$, Ewa Majchrzak ${ }^{2}$, Mateusz Duda ${ }^{1}$ \\ ${ }^{1}$ Higher School of Labour Safety Management in Katowice, Poland \\ ${ }^{2}$ Silesian University of Technology, Gliwice, Poland \\ bmochnacki@wszop.edu.pl
}

\begin{abstract}
In the paper the simplified model of thermal processes proceeding in the domain of biological tissue secured with protective clothing is discussed. In particular, the simplification of the mathematical model consists in the omission of the real layer of fabric for which the transient temperature field is determined by the Fourier equation and the introduction in this place of the additional thermal resistance appearing in the boundary condition determining the heat exchange between tissue and environment. In this way both the mathematical model of the thermal processes in the system considered and also numerical realization are greatly simplified. To verify the effectiveness of the approach proposed, the solution of the basic problem and the simplified one have been solved (1D task) using the finite difference method and the results have been compared. It turned out that the results are close and from the practical point of view such simplification is fully acceptable.
\end{abstract}

Keywords: bioheat transfer, protective clothing, modeling of thermal processes, finite difference method

\section{Introduction}

The aim of considerations is the numerical analysis of thermal processes proceeding in the system external heat source - protective clothing - air gap - skin tissue. The mathematical model of the process is created by a system of PDE, in particular the non-steady temperature field in the domain of skin tissue is described by the Pennes equation [1], while the temperature distribution in the fabric - by the wellknown Fourier equation. The model is supplemented by the boundary conditions given on the external surface of the fabric, between the fabric and skin tissue (the air gap should be taken into account), between tissue sub-domains and also on the surface conventionally limiting the tissue domain. The initial distribution of temperature is also assumed to be known.

This rather complex problem can be solved using numerical methods and the authors have the results of simulations concerning the above formulated boundaryinitial problem. The interesting task related to the problem discussed is the comparison of the solution obtained with the solution of simplified model in which the heat flow between skin tissue and environment is found without the real fabric 
layer. In this place the substitute thermal resistance resulting from the serial connection of partial resistances (environment, fabric, air gap) can be introduced. Next, on the external surface of skin tissue the Robin condition can be formulated (the heat transfer coefficient is the inverse of substitute thermal resistance). At the stage of numerical computations the approach discussed is essentially simpler. The testing computations (the finite difference method for the 1D task has been applied) show that the simplification proposed is fully acceptable.

\section{Governing equations}

We consider the non-homogeneous domain being the composition of the skin tissue, the layer of protective clothing and the air gap between skin and fabric, at the same time we assume that the material from which the clothing is made can be treated as a homogeneous one.

The temperature field in the tissue sub-domain is described by the following energy equation $[1,2]$ (the $1 \mathrm{D}$ problem is considered and taking into account the real geometrical conditions, e.g. chest, back, such a simplification is quite acceptable):

$$
x \in \Omega_{e}: \quad C_{e} \frac{\partial T_{e}(x, t)}{\partial t}=\lambda_{e} \frac{\partial^{2} T_{e}(x, t)}{\partial x^{2}}+G_{B e} C_{B}\left[T_{B}-T_{e}(x, t)\right]+Q_{m e t, e}
$$

where $e=1,2,3$ corresponds to skin tissue sub-domains (epidermis, dermis, subcutaneous region), $C_{e}$ is the volumetric specific heat of $\Omega_{e}, \lambda_{e}$ is the thermal conductivity, $G_{B e}$ is the perfusion coefficient, $C_{B}$ is the volumetric specific heat of blood, $Q_{m e t, e}$ is the metabolic heat source. It should be pointed out that the second component on the right-hand side of equation (1) corresponds to the so-called perfusion heat source and it is connected with the presence of the big number of capillaries uniformly distributed in the domain of soft tissue.

The temperature distribution in the fabric sub-domain results from the wellknown Fourier equation

$$
x \in \Omega_{F}: \quad C_{F} \frac{\partial T_{F}(x, t)}{\partial t}=\lambda_{F} \frac{\partial^{2} T_{F}(x, t)}{\partial x^{2}}
$$

where $C_{F}$ is the volumetric specific heat and $\lambda_{F}$ is the thermal conductivity of fabric. One can see that in equations (1) and (2), the constant values of specific heats and thermal conductivities are assumed (the numerical data quoted in literature correspond, as a rule, to the constant numbers - e.g. [3]).

On the external surface of the fabric the Robin condition is given

$$
x=0: \quad q_{F 0}(x, t)=\alpha_{s}\left[T_{F}(x, t)-T_{a}\right]
$$

where $\alpha_{s}$ is the heat transfer coefficient, while $T_{a}$ is the ambient temperature. 
Between the fabric and tissue the thermal resistance $R(t)$ of the air gap is defined as the result of parallel connection of the radial and conductional components, in particular

$$
\alpha_{r}(t)=C_{c} \varepsilon_{F-T} 10^{-4}\left[T_{b F}(t)+T_{b T}(t)\right]\left[\left(\frac{T_{b F}(t)}{100}\right)^{2}+\left(\frac{T_{b T}(t)}{100}\right)^{2}\right], \quad R_{r}(t)=\frac{1}{\alpha_{r}(t)}
$$

where $C_{c}$ is the Stefan-Boltzmann constant, $\varepsilon_{F-T}$ is the substitute emissivity of the fabric - tissue system, $T_{b F}, T_{b T}[\mathrm{~K}]$ denote the fabric and tissue boundary temperatures. The conductional thermal resistance equals

$$
R_{c}(t)=R_{c}=\frac{\delta_{a}}{\lambda_{a}}
$$

where $\delta_{a}$ is the air gap thickness, $\lambda_{a}$ is the air thermal conductivity. The appropriate boundary condition is of the form

$$
x \in \Gamma_{F-T}: \quad q_{F}(x, t)=\frac{T_{b F}(x, t)-T_{b T}(x, t)}{R(t)}=q(x, t)
$$

or

$$
x \in \Gamma_{F-T}:\left\{\begin{array}{c}
q_{F}(x, t)=q(x, t) \\
T(x, t)=T_{F}(x, t)-R(t) q(x, t)
\end{array}\right.
$$

The convectional heat transfer in the gap can be neglected (a 'trapped' air).

Between skin tissue sub-domains the ideal thermal contact is assumed (continuity of the temperature and heat fluxes), while at the point corresponding to the right edge of the domain one has

$$
T_{3}(x, t)=T_{\text {in }}
$$

where $T_{\text {in }}$ is the temperature of body interior.

The mathematical model should be supplemented by the initial conditions. In the domain of fabric and tissue the initial temperatures are given, in particular

$$
t=0: \quad T_{F}(x, 0)=T_{F 0}
$$

while the initial condition for the tissue is the following:

$$
t=0: \quad T(x, 0)=T_{0}(x)
$$

and the distribution of $T_{0}(x)$ is assumed in the form of quadratic function [3]. 
The simplified model is created by the equations (1), boundary conditions in the form (3) (co-ordinate $x=0$ corresponds to the skin surface), continuity conditions between tissue sub-domains and conditions (8), (10). The heat transfer coefficient $\alpha$ in the case considered is the inverse of substitute thermal resistance given by the formula

$$
R_{s}=\frac{1}{\alpha_{s}}+\frac{\delta_{F}}{\lambda_{F}}+\frac{R_{c} R_{r}}{R_{c}+R_{r}}
$$

where $\alpha_{s}$ is the heat transfer coefficient (exactly the same as in the condition (3)), $\delta_{F}$ is the fabric thickness, $\lambda_{F}$ is the fabric thermal conductivity, $R$ (as previously) results from the parallel connection of $R_{c}$ and $R_{r}(t)$ (equations (4) and (5)), but $R_{r}(t)$ is treated as the constant value. The results of basic problem solution show that this assumption is quite acceptable. Summing up, the resistance $R$ results from the series-parallel connection of convectional, radial and conductional thermal resistances.

\section{Finite difference algorithm}

Let us introduce the time grid with the constant step $\Delta t=t^{f}-t^{f-1}$ and the 1D spatial lattice with the constant step $h$. The 'boundary nodes' are located at the distance $0.5 h$ from the real boundary. Such a way of the grid construction improves the accuracy of the FDM scheme [4]. Using the explicit scheme of the FDM for the transition $t^{f-1} \rightarrow t^{f}, f=1,2, \ldots, F$, one obtains the following approximation of equation (1) corresponding to the node $x_{i}$ [4]

$$
C_{i} \frac{T_{i}^{f}-T_{i}^{f-1}}{\Delta t}=\frac{T_{i+1}^{f-1}-T_{i}^{f-1}}{R_{i+1}} \Phi_{i+1}+\frac{T_{i-1}^{f-1}-T_{i}^{f-1}}{R_{i-1}} \Phi_{i-1}+G_{B i} C_{B}\left[T_{B}-T_{i}^{f-1}\right]+Q_{m e t, i}
$$

where $\Phi_{i-1}=\Phi_{i+1}=1 / \mathrm{h}$ are the mesh shape functions (in the case of Cartesian coordinate system), $R_{i-1}, R_{i+1}$ are the thermal resistances in directions $i+1$ and $i-1$. In the case of node for which the adjacent nodes belong to the tissue sub-domain the following formulas should be used:

$$
R_{i+1}=\frac{0.5 h}{\lambda_{i}}+\frac{0.5 h}{\lambda_{i+1}}, \quad R_{i-1}=\frac{0.5 h}{\lambda_{i}}+\frac{0.5 h}{\lambda_{i-1}}
$$

For the node near the boundary between tissue and air gap one has

$$
R_{i-1}=\frac{0.5 h}{\lambda_{i}}+\frac{0.5 h}{\lambda_{i-1}}+R\left(t^{f-1}\right), \quad R_{i-1}=\frac{0.5 h}{\lambda_{i}}+R_{s}
$$


The first formula corresponds to the basic model, while the second corresponds to the simplified one (then in place of $T_{i-1}^{f-1}$ one should introduce the ambient temperature $T_{a}$ ).

The basic model requires the construction of FDM equations for the fabric sub-domain. These equations are essentially simpler and they will not be discussed here. Because the explicit scheme is applied, the stability condition therefore must be fulfilled. In the case considered the limitation of time step results from the inequality

$$
1-\frac{\Delta t}{C_{i}}\left(\frac{\Phi_{i+1}}{R_{i+1}}+\frac{\Phi_{i-1}}{R_{i-1}}+G_{B i} C_{B}\right) \geq 0
$$

\section{Example of computations}

The numerical computations concern both the basic model and the simplified one. Thermophysical parameters of skin tissue and the thicknesses of the epidermis and dermis are taken from [5]. Additionally, the thickness of fabric equals $6.67 \mathrm{~mm}$ and $C_{F}=0.24 \mathrm{MJ} /\left(\mathrm{m}^{3} \mathrm{~K}\right), \lambda_{F}=0.17 \mathrm{~W} /(\mathrm{mK})$. The total thickness of the tissue sub-domain is equal to $12 \mathrm{~mm}$, while the width of air gap $\delta_{a}=5 \mathrm{~mm}$. The thermal resistance (simplified model) $R_{s}=0.078 \mathrm{~m}^{2} \mathrm{~K} / \mathrm{W}$. On the external surface of the fabric (basic model) $\alpha_{s}=100 \mathrm{~W} /\left(\mathrm{m}^{2} \mathrm{~K}\right)$ and $T_{a}=60^{\circ} \mathrm{C}$. The blood temperature $T_{B}=T_{\text {in }}=37^{\circ} \mathrm{C}$.

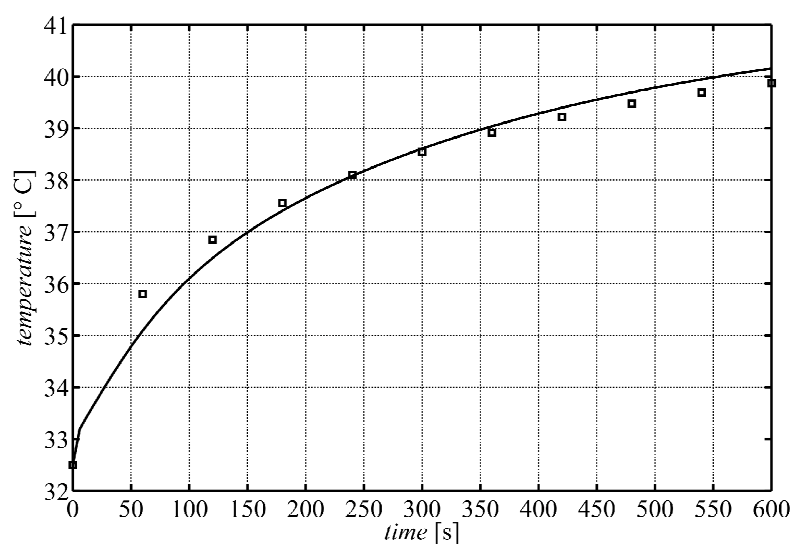

Fig. 1. Temperature history at the point corresponding to skin surface (symbols - simplified model)

In Figures 1 and 2 the results of the computations are presented. In particular (Fig. 1), the heating curve at the point corresponding to the skin surface is shown. The next figure shows the temperature profiles in the tissue sub-domain for times 
3, 6 and 9 min. The solution of the basic model and the simplified one are close. The rather small differences result from the averaging of thermal resistance between fabric and skin and due to the omission of thermal process dynamics in the fabric sub-domain. From the practical point of view, the solution of simplified model is quite acceptable.

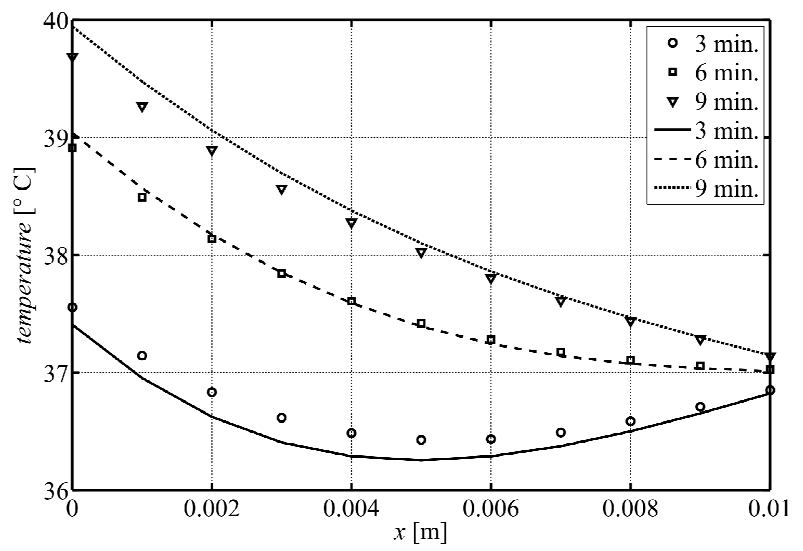

Fig. 2. Temperature profiles (symbols - simplified model)

\section{Acknowledgement}

The paper is a part of Research Project PB3/2013 sponsored by WSZOP Katowice.

\section{References}

[1] Pennes H.H., Analysis of tissue and arterial blood temperatures in the resting human forearm, Journal of Applied Physiology 1948, 1, 93-122.

[2] Ciesielski M., Mochnacki B., Numerical simulation of the heating process in the domain of tissue insulated by protective clothing, Journal of Applied Mathematics and Computational Mechanics 2014, 13, 2, 13-20.

[3] Majchrzak E., Mochnacki B., Jasiński M., Numerical modelling of bioheat transfer in multilayer skin tissue domain subjected to a flash fire, Computational Fluid and Solid Mechanics 2003, II, 1766-1770.

[4] Mochnacki B., Suchy J.S., Numerical Methods in Computations of Foundry Processes, PFTA, Cracow 1996.

[5] Majchrzak E., Chapter 3, [in:] Technical Mechanics 12, R. Bedzinski (ed.), IPPT, Warsaw 2011. 